\title{
Travelling Waves in Near-Degenerate Bistable Competition Models
}

\author{
E.O. Alzahrani, F.A. Davidson* and N. Dodds
}

Division of Mathematics, Dundee University, Dundee DD1 4HN, Scotland UK.

\begin{abstract}
We study a class of bistable reaction-diffusion systems used to model two competing species. Systems in this class possess two uniform stable steady states representing semi-trivial solutions. Principally, we are interested in the case where the ratio of the diffusion coefficients is small, i.e. in the near-degenerate case. First, limiting arguments are presented to relate solutions to such systems to those of the degenerate case where one species is assumed not to diffuse. We then consider travelling wave solutions that connect the two stable semi-trivial states of the nondegenerate system. Next, a general energy function for the full system is introduced. Using this and the limiting arguments, we are able to determine the wave direction for small diffusion coefficient ratios. The results obtained only require knowledge of the system kinetics.
\end{abstract}

Key words: competition, reaction-diffusion, free energy, bistable, travelling waves

AMS subject classification: 35K57, 35C07, 35B65

\section{Introduction}

We are interested in component-wise monotone travelling wave solutions of the system of equations

$$
\begin{gathered}
u_{t}=u_{x x}+f(u, v), \\
v_{t}=\epsilon^{2} v_{x x}+g(u, v),
\end{gathered}
$$

for $(x, t) \in \mathbb{R} \times \mathbb{R}^{+}$for which the asymptotic conditions

$$
(u, v)(-\infty, t)=S_{-}, \quad(u, v)(\infty, t)=S_{+}, \quad t>0
$$

\footnotetext{
*Corresponding author. E-mail: fdavidso@maths.dundee.ac.uk
} 
are satisfied. The literature concerning the analysis and applications of systems of the form (1.1) is now vast and travelling wave solutions have been studied for many decades see e.g. [1, 3, 13, 14, $15,17]$ and the references therein. One well-studied application has been to population dynamics where the components $u, v$ are assumed to be interacting species. The term "species" is used in its loosest sense here and can be taken to represent different species of animals, microbes or plants, chemical species, cell types etc. The species are assumed to move in a random manner (modelled via the diffusion term). The ratio of the diffusion coefficients, denoted by $\epsilon^{2}$, is not necessarily small, but in this paper we are mainly interested in the case where $\epsilon \rightarrow 0$. This represents the case where the mobility of one species is relatively much greater than that of the other. The interaction of the species occurs through the functions $f, g$ and can be synergistic, of predator-prey type or competitive. Here, we focus on the last of these, where competition between the species is for a common resource. In $[5,6]$ the competition is taken to be between normal and malignant cells, where the common resource can be considered to be oxygen. It is assumed that normal cells denoted here by $v$ diffuse little, where as the malignant cells, denoted here by $u$, are taken to be highly motile.

To be precise regarding the interactions, we make the following assumptions on $f$ and $g$ (see [5]). Here and below, we will employ the following notation. The pair $(u, v)$ will be used to represent a specific solution. However, for ease of notation, if $K: \mathbb{R}^{2} \rightarrow \mathbb{R}$ is any sufficiently differentiable function, then $K_{u}$ and $K_{v}$ will denote the partial derivatives of $K$ with respect to the first and second variables, respectively. Also, we use the notation $|\cdot|_{i}$ to represent the standard uniform norm associated with $C^{i}(\mathbb{R}), i=0,1,2$.

Assumption 1. The non-linearities $f, g \in C^{2}\left([0,1]^{2}, \mathbb{R}\right)$ satisfy:

(1) $f(0, v)=0=g(u, 0)$.

(2) (1.1) has exactly two stable, uniform equilibria $S_{-}=(0,1)$ and $S_{+}=(1,0)$ and two unstable, uniform equilibria $(0,0)$ and $\left(u_{s}, v_{s}\right)$.

(3) $f_{v}(u, v)<0, g_{u}(u, v)<0$ for $(u, v) \in(0,1)^{2}$.

(4) The non-trivial solutions $(u, v)$ of $g(u, v)=0$ are given by $u=\Gamma(v)$ for a monotonically decreasing function $\Gamma$. Setting $\Gamma(1)=0$ and $\Gamma(0)=\hat{u}$, where $0<\hat{u}<1, \Gamma$ has an inverse $\hat{\gamma} \in C^{1}([0, \hat{u}],[0,1])$, which can be extended trivially to a function $\gamma \in C^{0}([0,1],[0,1])$ where

$$
\gamma(u)= \begin{cases}\hat{\gamma} & u \in[0, \hat{u}] \\ 0 & u \in(\hat{u}, 1]\end{cases}
$$

Remark 2. In Section 4, we discuss how condition (4) of Assumption 1 can be relaxed to include a class of non-monotone functions $\mathrm{g}$. However, for ease of exposition we provide a detailed account of the monotone case first.

Stability, as required by condition (2), is equivalent to both the eigenvalues of the associated Jacobian having negative real parts when evaluated at the equilibrium point. (Instability is defined accordingly.) An illustration of typical nullclines is given in Figure 1.

For future reference we call the following definition of $f$ and $g$, the "classic Lotka-Volterra" terms and denote these by CLV: 


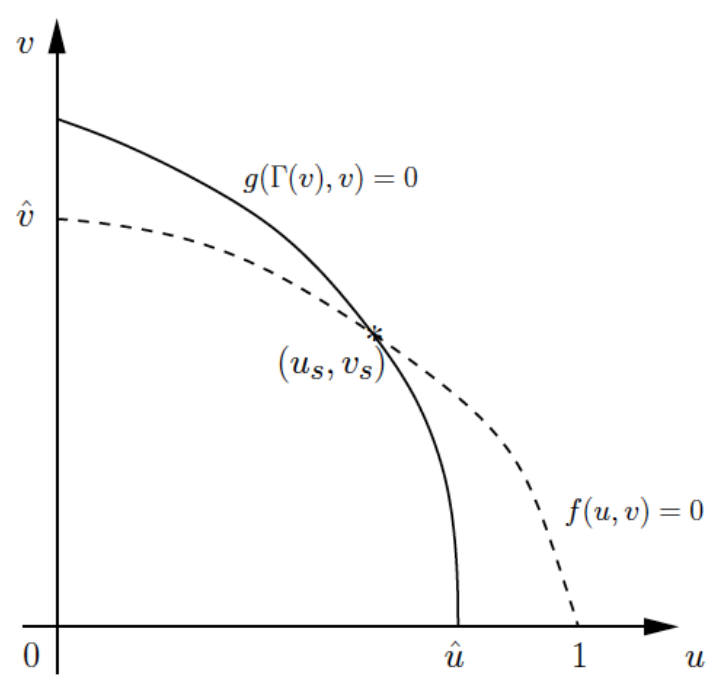

Figure 1: Schematic representation of the nullclines $f$ and $g$ satisfying Assumption 1.

$$
f(u, v)=u(1-u-\alpha v), \quad g(u, v)=\delta v(1-v-\beta u),
$$

where $\alpha, \beta$ and $\delta$ are positive constants. It is straightforward to show that the conditions of Assumption 1.1 are satisfied by the CLV terms, provided the inter-specific competition rates satisfy $\alpha, \beta>1$. Note also that in this case the nullclines shown in Figure 1 are simply straight lines and the points of intersection $\hat{u}$ and $\hat{v}$ are equal to $1 / \beta$ and $1 / \alpha$, respectively.

As is standard, by first setting $z=x-c t$ for some constant $c$, a travelling wave solution of (1.1), (1.2) is defined to be a function $(u(z), v(z)) \in\left[C^{2}(\mathbb{R})\right]^{2}$ that satisfies

$$
\begin{aligned}
-c u^{\prime} & =u^{\prime \prime}+f(u, v) \\
-c v^{\prime} & =\epsilon^{2} v^{\prime \prime}+g(u, v) \\
(u, v)(-\infty) & =S_{-}, \quad(u, v)(\infty)=S_{+} .
\end{aligned}
$$

If $c>0$ then such a solution represents a wave front moving to the right in the $x$-frame. Using the change of variables $w=1-u,(1.3)$ becomes

$$
\begin{aligned}
-c w^{\prime} & =w^{\prime \prime}-f(1-w, v):=w^{\prime \prime}+F(w, v), \\
-c v^{\prime} & =\epsilon^{2} v^{\prime \prime}+g(1-w, v):=\epsilon^{2} v^{\prime \prime}+G(w, v), \\
(w, v)(-\infty) & =(1,1), \quad(w, v)(\infty)=(0,0) .
\end{aligned}
$$

It follows from Assumption 1 that the monotonicity conditions $F_{v} \geq 0, G_{w} \geq 0$ hold and therefore we may directly apply Theorem 1.1 on p155 in [17] to ensure the existence of a monotone solution to (1.4). Thus a (component-wise) monotone solution of (1.3) exists corresponding to a unique 
(up to translation) travelling wave for (1.1), (1.2). Moreover, by constructing appropriate sub and supersolutions to (1.3) similar to those introduced in [6] and using essentially identical arguments, it can be shown that

$$
-K \leq c \leq L \epsilon,
$$

where $K$ and $L$ are positive constants independent of $\epsilon$. One immediate consequence of this result is that in the limit $\epsilon \rightarrow 0$ only left travelling waves exist.

The aim of this paper is to develop an understanding of the relationship between the solutions of (1.3) and those of the reduced or degenerate problem

$$
\begin{aligned}
-c u^{\prime} & =u^{\prime \prime}+f(u, v), \\
-c v^{\prime} & =g(u, v), \\
(u, v)(-\infty) & =S_{-}, \quad(u, v)(\infty)=S_{+} .
\end{aligned}
$$

Given its singular nature, it is not surprising that the solutions to (1.6), denoted here by $\left(u_{0}, v_{0}, c_{0}\right)$, may have quite different regularity properties to those of $(1.3)$ (see $[5,6]$ ). Therefore, it is not obvious a priori how the solutions of (1.3) behave in the limit as $\epsilon \rightarrow 0$ or how any such limit is related to $\left(u_{0}, v_{0}, c_{0}\right)$. In the following we establish this connection.

Singular limit problems in systems of reaction-diffusion equations is a subject which has received considerable interest, particularly using methods of singular perturbation theory (see e.g. $[7,8]$ and the references therein). However, these papers have, in general, focused on the construction of solutions via asymptotic expansions. Here, we focus on the regularity of the solutions in the limit. Regularity of solutions of such limiting problems has again attracted considerable, previous interest and most relevant to the work presented here are the methods for dealing with partially degenerate equations as developed by $[9,10,11]$ (see also $[4,16]$ ). The systems of equations studied in these latter papers are somewhat different to those considered here and contain kinetics of a different type. Hence, although some of the techniques developed in these papers can be used in the following, the nature of the kinetics studied here necessitates the development of alternative methods of proof. In particular, properties of the kinetics studied in $[9,10,11]$ allow for certain bounds on the solutions $\left(|u|_{2}\right.$ and $|v|_{2}$ here) to be developed independently of the wave speed $c$. This method of proof does not, in general, transfer to the class of systems studied here.

Establishing the regularity of solutions to (1.3) in the limit and hence their relationship to the solutions of (1.6) allows for the development of the energy arguments constructed in [5] and [6] to the general case $\epsilon>0$. These results are necessary in order to establish the direction of the travelling waves as is now discussed.

For scalar bistable reaction-diffusion equations, it is well-known that the sign of the wave speed is determined by the sign of the integral of the reaction function over a certain interval. This integral forms part of an energy function for the scalar equation (see e.g. p175 et seq. in [2]). For systems of bistable equations, the situation is far more complex and to date, no general energy function has been derived. However, in [5, 6] a potential for the reduced system (1.6) was introduced as follows. Suppose $\left(u_{0}, v_{0}\right)$ is a solution of (1.6), then define the function $h\left(u_{0}, v_{0}\right)$ by

$$
\left[h\left(u_{0}, v_{0}\right)\right](z)=-\int_{0}^{u_{0}(z)} f\left(\sigma, v_{0}(z)\right) d \sigma-\int_{v_{0}(z)}^{1} \int_{0}^{\Gamma(\tau)} f_{v}(\sigma, \tau) d \sigma d \tau, \quad z \in \mathbb{R}
$$


where, as detailed above, $\Gamma$ is given by $g(\Gamma(\tau), \tau)=0$, for $0<\tau<1$. After a little manipulation, it can be shown that $[h(1,0)](z) \equiv h\left(S_{+}\right)$where

$$
h\left(S_{+}\right)=-\left(\int_{0}^{\hat{u}} f(\sigma, \hat{\gamma}(\sigma)) d \sigma+\int_{\hat{u}}^{1} f(\sigma, 0) d \sigma\right)=-\int_{0}^{1} f(\sigma, \gamma(\sigma)) d \sigma
$$

and more easily that $[h(0,1)](z) \equiv h\left(S_{-}\right)=0$. It is shown in [6] that the sign of $h\left(S_{+}\right)-h\left(S_{-}\right)=h\left(S_{+}\right)$determines the direction of the wave and the regularity of the solution. A key point here is that for any given kinetics $f$ and $g$, the potential $h\left(S_{+}\right)$can be directly computed without knowledge of the solutions. To assist the reader, we include the key results concerning such solutions, that will be referenced below. The proofs can be found in [5] and [6].

Theorem 3. Suppose $f$ and $g$ satisfy Assumption 1.

(i) If $h\left(S_{+}\right)<0$, then there exists a monotone solution $\left(u_{0}, v_{0}, c_{0}\right)$ to (1.6) with $c_{0}<0$ and $\left(u_{0}, v_{0}\right) \in C^{2}(\mathbb{R}) \times C^{1}(\mathbb{R})$. If $c_{0}>0$, then no monotone solution to (1.6) exists.

(ii) If $h\left(S_{+}\right) \geq 0$, then there exists a monotone solution $\left(u_{0}, v_{0}, c_{0}\right)$ to (1.6) with $c_{0}=0$. In this case, $v_{0}$ has a unique singularity at $z^{*}$, say, and $u_{0}(z)=\Gamma\left(v_{0}\right)(z)$ for $z \leq z^{*}$ and $v_{0} \equiv 0$ otherwise. Hence, whilst $u_{0} \in C^{1}(\mathbb{R}), v_{0}$ is only continuous if $h\left(S_{+}\right)=0$ and only bounded if $h\left(S_{+}\right)>0$. However, away from this point, $\left(u_{0}, v_{0}\right)$ is in $\left[C^{2}\left(\mathbb{R} \backslash z^{*}\right)\right]^{2}$. If $c_{0} \neq 0$, then no monotone solution to (1.6) exists.

In both cases, these solutions correspond to monotone travelling waves for (1.1), (1.2) with $\epsilon=0$, that are unique up to translation.

A schematic representation of the $c_{0}=0$ solution is given in Figure 2. The singularity is at the point $\left(u^{*}, v^{*}\right)=:\left(u_{0}\left(z^{*}\right), v_{0}\left(z^{*}\right)\right)$.

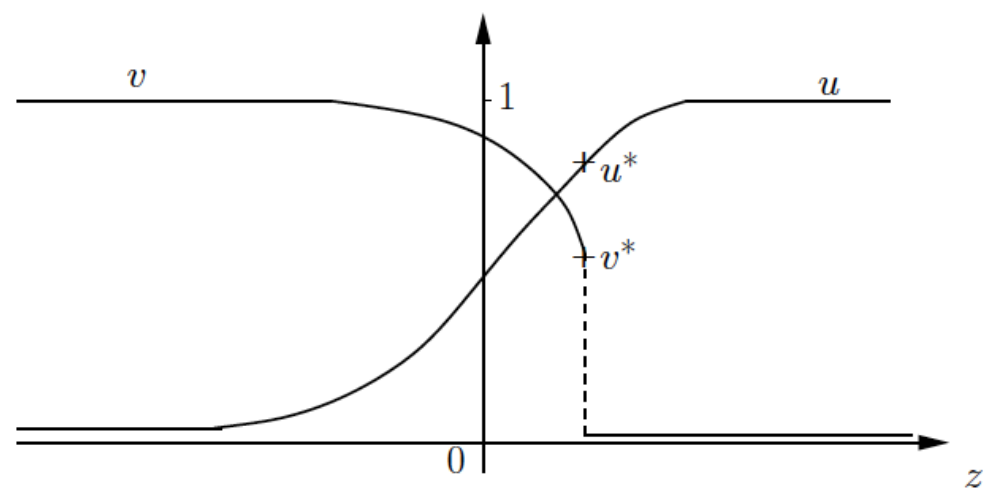

Figure 2: Schematic representation of the $c_{0}=0$ solution to the reduced problem (1.6).

Unfortunately, it is straightforward to show that (1.7) does not form a suitable potential for the more general problem (1.3). In this paper we construct an alternative and relate it to the definition 
(1.7). Thus, at least for $\epsilon$ sufficiently small, we show that the direction of travelling wave solutions for the general competitive bistable system (1.1) can be determined directly from the kinetics of the problem.

In Section 2 we construct arguments that determine the regularity of solutions to (1.3) in the limit as $\epsilon \rightarrow 0$. A particular problem to overcome is that despite remaining monotone in the limit, it is not clear whether one can a priori rule out that solutions "ripple" arbitrarily fast, i.e. their second derivatives change sign in a highly oscillatory manner. In fact, we establish that this cannot happen. In Section 3 we construct a free energy function for the general problem (1.3) and show how it can be used to determine the sign of $c$ for any $\epsilon>0$. Finally, using the convergence results developed in Section 2, the relationship between this new, general free energy function and the function given in (1.7) for the reduced problem is established.

\section{Convergence in the limit $\epsilon \rightarrow 0$}

The main result of this section is as follows.

Theorem 4. Suppose that $\left(u_{\epsilon}, v_{\epsilon}, c_{\epsilon}\right)$ is a classical component-wise monotone solution of (1.3) i.e. $u_{\epsilon}, v_{\epsilon} \in C^{2}(\mathbb{R}), u_{\epsilon}^{\prime}(z)>0>v_{\epsilon}^{\prime}(z)$ for $z \in \mathbb{R}$. Let $\left(u_{0}, v_{0}, c_{0}\right)$ denote the monotone solution of (1.6) as detailed above. Then we have the following convergence results.

(i) If $\lim _{\epsilon \rightarrow 0} c_{\epsilon} \neq 0$, then after suitable translation, $\left(u_{\epsilon}, v_{\epsilon}\right) \rightarrow\left(u_{0}, v_{0}\right)$ uniformly on any closed interval of $\mathbb{R}$.

(ii) If $\lim _{\epsilon \rightarrow 0} c_{\epsilon}=0$, then after suitable translation, $\left(u_{\epsilon}, v_{\epsilon}\right) \rightarrow\left(u_{0}, v_{0}\right)$ where the convergence is uniform on any closed interval in $\mathbb{R} \backslash z^{*}$.

Remark 5. Many of the following results, which are used to prove Theorem 4, can be extended to hold on any compact subset of $\mathbb{R}$. In particular, statement ( $i)$ of Theorem 4 holds in this more general case.

The proof requires a series of results, some of which appear in part in [6]. We fill some apparent gaps in the proofs given there and in doing so derive statements more useful for our purposes.

We now set about proving Theorem 4 by considering sequences of solutions, $\left(u_{k}, v_{k}, \epsilon_{k}, c_{k}\right) \in$ $C^{2}(\mathbb{R}) \times C^{2}(\mathbb{R}) \times \mathbb{R} \times \mathbb{R}, k=1,2, \ldots$ of (1.3) such that $\epsilon_{k} \rightarrow 0$ as $k \rightarrow \infty$. Solutions of system (1.3) are invariant to translations in $z$ and so we impose a normalisation to the sequence $\left(u_{k}, v_{k}, \epsilon_{k}, c_{k}\right)$ by setting $u_{k}(0)=u_{s}$ for $k \in \mathbb{N}$. (Of course, this normalisation does not a priori fix the value of $v_{k}(0)$, a point that will be important later.) The cases of Theorem 4 will be dealt with separately. Clearly, for any sequence $\left\{c_{k}\right\}$, there exists a subsequence which either tends to zero or is bounded away from zero. If necessary, the results below refer to such subsequences. 


\subsection{Case (i): $\lim _{k \rightarrow \infty} c_{k} \neq 0$.}

Lemma 6. If $\lim _{k \rightarrow \infty} c_{k} \neq 0$, then there exists a positive constant $K_{1}$ independent of $k$ such that

$$
\left|u_{k}\right|_{2}+\left|v_{k}\right|_{2} \leq K_{1}
$$

Proof. As only component-wise monotone solutions $\left(u_{k}, v_{k}\right) \in[0,1] \times[0,1]$ are being considered here, it follows immediately that $\left|u_{k}\right|_{0} \leq 1$ and $\left|v_{k}\right|_{0} \leq 1, \forall k \in \mathbb{N}$. Thus, and by the continuity of $f$ and $g, \exists C_{1}, C_{2}>0$ such that

$$
\left|f\left(u_{k}(z), v_{k}(z)\right)\right| \leq C_{1} \quad \forall z \in \mathbb{R} \quad \forall k \in \mathbb{N}
$$

and

$$
\left|g\left(u_{k}(z), v_{k}(z)\right)\right| \leq C_{2} \quad \forall z \in \mathbb{R} \quad \forall k \in \mathbb{N} .
$$

For $k$ fixed, suppose that $\left|u_{k}^{\prime}(z)\right|$ obtains its maximum value at $z=z_{0}$, say. Then $u_{k}^{\prime \prime}\left(z_{0}\right)=0$ and from (1.3), it follows that for all $z \in \mathbb{R}$,

$$
\left|c_{k} u_{k}^{\prime}(z)\right| \leq\left|c_{k} u_{k}^{\prime}\left(z_{0}\right)\right|=\left|f\left(u_{k}\left(z_{0}\right), v_{k}\left(z_{0}\right)\right)\right| \leq \max _{(u, v) \in[0,1] \times[0,1]}|f(u, v)| \leq C_{1} .
$$

As $\left|c_{k}\right|>K>0$ for all $k$, then a bound on $\left|u_{k}^{\prime}\right|_{0}$ that is independent of $k$ follows. A bound for $\left|v_{k}^{\prime}\right|_{0}$ can be found analogously. Thus a bound for $\left|u_{k}^{\prime \prime}\right|_{0}$ can be obtained directly from (1.3). Finally, as $g \in C^{2}$, it follows from (1.3) that $v_{k} \in C^{3}(\mathbb{R})$ and so we can differentiate the second equation in (1.3) wrt $z$. Then, noting that if $\left|v_{k}^{\prime \prime}(z)\right|$ attains its maximum value at $z=z_{0}$, say, then $v_{k}^{\prime \prime \prime}\left(z_{0}\right)=0$ and we obtain

$$
-c_{k} v_{k}^{\prime \prime}\left(z_{0}\right)=g^{\prime}\left(u_{k}\left(z_{0}\right), v_{k}\left(z_{0}\right)\right)=g_{u}\left(u_{k}\left(z_{0}\right), v_{k}\left(z_{0}\right)\right) u_{k}^{\prime}\left(z_{0}\right)+g_{v}\left(u_{k}\left(z_{0}\right), v_{k}\left(z_{0}\right)\right) v_{k}^{\prime}\left(z_{0}\right) .
$$

From the previous bounds, the smoothness of $g$ and again using the assumption $\left|c_{k}\right|>K>0$, a bound for $\left|v_{k}^{\prime \prime}\right|_{0}$ follows as required.

Lemma 7. Let $\mathcal{D}$ be any closed interval in $\mathbb{R}$, let $\alpha \in(0,1)$ and suppose $\left|c_{k}\right|>K>0$ for some constant $K$. Then $\exists\left(u^{*}, v^{*}\right) \in C^{2}(\mathcal{D}) \times C^{1, \alpha}(\mathcal{D})$ and a subsequence $\left\{\left(u_{k_{l}}, v_{k_{l}}\right)\right\}_{l=1}^{\infty}$ of $\left\{\left(u_{k}, v_{k}\right)\right\}_{k=1}^{\infty}$ such that

$$
\left(u_{k_{l}}, v_{k_{l}}\right) \rightarrow\left(u^{*}, v^{*}\right) \quad \text { in } \quad C^{2}(\mathcal{D}) \times C^{1, \alpha}(\mathcal{D}) .
$$

Proof. By Lemma 6, it follows that $\left\{u_{k}\right\}$ and $\left\{v_{k}\right\}$ are bounded in $C^{2}(\mathcal{D})$. Since

$$
C^{2}(\mathcal{D}) \subset \subset C^{1, \alpha}(\mathcal{D})
$$

for any $\alpha \in(0,1)$, it follows that

$$
\left(u_{k_{l}}, v_{k_{l}}\right) \rightarrow\left(u^{*}, v^{*}\right) \quad \text { in } \quad\left[C^{1, \alpha}(\mathcal{D})\right]^{2} .
$$


Moreover, the $u$-equation in (1.3) can be rewritten as

$$
u_{k}^{\prime \prime}=-c_{k} u_{k}^{\prime}-f\left(u_{k}, v_{k}\right) .
$$

By Lemma 6, the boundedness of $\left\{c_{k}\right\}$ given by (1.5), and the continuity of $f$, a (sub)sequence of the RHS of (2.2) converges in $C^{0}(\mathcal{D})$. Therefore $\exists U^{*} \in C^{0}(\mathcal{D})$ such that $u_{k_{l_{j}}}^{\prime \prime} \rightarrow U^{*} \in C^{0}(\mathcal{D})$. Taking any two elements of $\left\{u_{k_{l_{j}}}\right\}, u_{i}$ and $u_{j}$, say, we have

$$
\begin{aligned}
\left|u_{i}-u_{j}\right|_{\mathcal{D}, 2}= & \sup _{\mathcal{D}}\left|u_{i}-u_{j}\right|+\sup _{\mathcal{D}}\left|u_{i}^{\prime}-u_{j}^{\prime}\right|+\sup _{\mathcal{D}}\left|u_{i}^{\prime \prime}-u_{j}^{\prime \prime}\right| \\
\leq & \sup _{\mathcal{D}}\left|u_{i}-u^{*}\right|+\sup _{\mathcal{D}}\left|u^{*}-u_{j}\right|+\sup _{\mathcal{D}}\left|u_{i}^{\prime}-u^{*^{\prime}}\right|+\sup _{\mathcal{D}}\left|u^{*^{\prime}}-u_{j}^{\prime}\right| \\
& +\sup _{\mathcal{D}}\left|u_{i}^{\prime \prime}-U^{*}\right|+\sup _{\mathcal{D}}\left|U^{*}-u_{j}^{\prime \prime}\right| \\
\rightarrow & 0 \quad \text { as } i, j \rightarrow \infty,
\end{aligned}
$$

i.e. $\left\{u_{k_{l_{j}}}\right\}$ is a Cauchy sequence in $C^{2}(\mathcal{D})$. Hence by the completeness of $C^{2}(\mathcal{D})$, it follows that $\exists U \in C^{2}(\mathcal{D})$ such that

$$
u_{k_{l_{j}}} \rightarrow U
$$

Hence,

$$
U=u^{*}, \quad U^{\prime}=u^{*^{\prime}} \quad \text { and } \quad U^{\prime \prime}=u^{*^{\prime \prime}},
$$

i.e. $u^{*} \in C^{2}(\mathcal{D})$.

We can now prove the first part of Theorem 4. The final step is to show that $\left(u^{*}, v^{*}\right)$ satisfies (1.6) in some sense.

Proof of Theorem $4(i)$. By Lemma 7, it follows that $\left(u^{*}, v^{*}\right) \in C^{2}(\mathcal{D}) \times C^{1}(\mathcal{D})$ for any closed interval $\mathcal{D} \subset \mathbb{R}$. Moreover, $\left(u^{*}, v^{*}\right)$ satisfies

$$
-c_{0} u^{*^{\prime}}=u^{*^{\prime \prime}}+f\left(u^{*}, v^{*}\right) .
$$

Also, since $\left|v_{k}^{\prime \prime}\right|_{0}$ is bounded, $\left|\epsilon_{k}^{2} v_{k}^{\prime \prime}\right|_{0} \rightarrow 0$ as $k \rightarrow \infty$, and it follows that $\left(u^{*}, v^{*}\right)$ satisfies

$$
-c_{0} v^{*^{\prime}}=g\left(u^{*}, v^{*}\right), \quad z \in \mathcal{D} .
$$

As this holds for any closed interval $\mathcal{D} \subset \mathbb{R}$, we are left to consider three possibilities: (i) $\left(u^{*}, v^{*}\right)=$ $\left(u_{0}, v_{0}\right)$, the monotone solution of (1.6); (ii) $v^{*} \equiv 0$ and $u^{*}$ is a strictly monotone function satisfying (1.6) with $v \equiv 0$, or (iii) $\left(u^{*}, v^{*}\right)=\left(u_{s}, v_{s}\right)$. Cases (ii) and (iii) can be dismissed using essentially identical arguments to those in the proof of Theorem 2.1 in [6]. Thus we have the desired result. 
2.2. Case (ii): $\lim _{k \rightarrow \infty} c_{k}=0$.

Lemma 8. If $\lim _{k \rightarrow \infty} c_{k}=0$, then there exists positive constants $K_{i}, i=2,3,4$ independent of $k$ such that

$$
\begin{aligned}
\left|u_{k}\right|_{2} & \leq K_{2}, \\
\left|v_{k}\right|_{0} & \leq 1 \\
\left|c_{k} v_{k}^{\prime}\right|_{0} & \leq K_{3} \\
\left|\epsilon_{k}^{2} v_{k}^{\prime \prime}\right|_{0} & \leq K_{4} .
\end{aligned}
$$

Moreover,

$$
\lim _{k \rightarrow \infty}\left|\epsilon_{k}^{2} v_{k}^{\prime}\right|_{0}=0
$$

Proof. The required bounds on $\left|u_{k}\right|_{0},\left|v_{k}\right|_{0},\left|c_{k} u_{k}^{\prime}\right|_{0}$ (and hence $\left|u_{k}^{\prime \prime}\right|_{0}$ ) and $\left|c_{k} v_{k}^{\prime}\right|_{0}$ (and hence $\left|\epsilon_{k}^{2} v_{k}^{\prime \prime}\right|_{0}$ ) follow from similar arguments to those used in the proof of Lemma 6. In particular,

$$
\left|u_{k}^{\prime \prime}\right|_{0} \leq K
$$

for some constant $K$ independent of $k$. As noted in [6], the bound on $\left|u_{k}^{\prime \prime}\right|_{0}$ can be used to generate a bound on $\left|u_{k}^{\prime}\right|$ independent of $c_{k}$ thus: for any $z, \hat{z} \in \mathbb{R}$,

$$
1>\left|u_{k}(\hat{z})-u_{k}(z)\right|=\left|(\hat{z}-z) u_{k}^{\prime}(z)+\int_{z}^{\hat{z}} \int_{z}^{\zeta} u_{k}^{\prime \prime}(\xi) \mathrm{d} \xi \mathrm{d} \zeta\right|
$$

and therefore using (2.8)

$$
\left|u_{k}^{\prime}(z)\right| \leq \inf _{\hat{z}}\left(|\hat{z}-z|^{-1}+|\hat{z}-z| K\right)=2 \sqrt{K}, \quad z \in \mathbb{R} .
$$

Hence, (2.3) holds.

Finally, let $q_{k}=\epsilon_{k}^{2} v_{k}$. Then applying the same reasoning as directly above, it follows from the second equation in (1.3) that for any $z, \hat{z} \in \mathbb{R}$

$$
\epsilon_{k}^{2}>\left|q_{k}(\hat{z})-q_{k}(z)\right|=\left|(\hat{z}-z) q_{k}^{\prime}(z)+\int_{z}^{\hat{z}} \int_{z}^{\zeta} q_{k}^{\prime \prime}(\xi) \mathrm{d} \xi \mathrm{d} \zeta\right| .
$$

As above and using (2.6) yields

$$
\left|q_{k}^{\prime}(z)\right| \leq \inf _{\hat{z}}\left(\epsilon_{k}^{2}|\hat{z}-z|^{-1}+K_{4}|\hat{z}-z|\right)=2 \epsilon_{k} \sqrt{K_{4}}, \quad z \in \mathbb{R}
$$

The result follows directly.

Since by assumption $c_{k} \rightarrow 0$ (and $\epsilon_{k} \rightarrow 0$ ) neither (2.5) nor (2.7) eliminate the possibility that $\left|v_{k}^{\prime}(z)\right| \rightarrow \infty$ as $k \rightarrow \infty$ at some possibly infinite number of points $z \in \mathbb{R}$. Consequently, in this case we cannot directly obtain convergence results analogous to Lemma 7. However, using these 
bounds and the monotonicity of solutions provides the desired result as will be shown shortly. First, by integrating the second equation in (1.3) over $\mathbb{R}$ and applying the appropriate limiting conditions on $v_{k}(z)$, it is straightforward to show that

$$
c_{k}=\int_{\mathbb{R}} g\left(u_{k}(z), v_{k}(z)\right) d z
$$

and hence in the case under consideration, $\int_{\mathbb{R}} g\left(u_{k}(z), v_{k}(z)\right) d z \rightarrow 0$ as $k \rightarrow \infty$. Using (2.7), we have the much stronger result that this convergence also holds over any closed interval.

Lemma 9. Let $[a, b] \subset \mathbb{R}$. Then

$$
\lim _{k \rightarrow \infty} \int_{a}^{b} g\left(u_{k}(z), v_{k}(z)\right) d z=0 .
$$

Proof. Take any $[a, b] \subset \mathbb{R}$. Then integrating the second equation in (1.3) over $[a, b]$ yields

$$
-c_{k}\left[v_{k}(b)-v_{k}(a)\right]=\epsilon_{k}^{2}\left[v_{k}^{\prime}(b)-v_{k}^{\prime}(a)\right]+\int_{a}^{b} g\left(u_{k}(z), v_{k}(z)\right) d z .
$$

Taking the limit as $k \rightarrow \infty$ and applying the bounds (2.4) and (2.7) yields the desired result.

Lemma 10. There exists at most one point $z^{*} \in \mathbb{R}$ such that $g\left(u_{k}(z), v_{k}(z)\right) \rightarrow 0$ uniformly on any closed interval of $\mathbb{R} \backslash z^{*}$.

Proof. Let $\mathcal{D} \subset \mathbb{R}$ be a closed interval. Then from Lemma 9 it follows directly that either $g\left(u_{k}(z), v_{k}(z)\right) \rightarrow 0$ uniformly on $\mathcal{D}$ or for each $k \in \mathbb{N}$, sufficiently large, there exists a number $\delta_{k}>0$ and three points $z_{-1}^{k}<z_{0}^{k}<z_{1}^{k} \in \mathcal{D}$ for which

$$
\left|z_{-1}^{k}-z_{1}^{k}\right|<\delta_{k}
$$

and

$$
\left|g\left(u_{k}\left(z_{-1}^{k}\right), v_{k}\left(z_{-1}^{k}\right)\right)\right|<\delta_{k}, \quad\left|g\left(u_{k}\left(z_{1}^{k}\right), v_{k}\left(z_{1}^{k}\right)\right)\right|<\delta_{k} \quad \text { and } \quad\left|g\left(u_{k}\left(z_{0}^{k}\right), v_{k}\left(z_{0}^{k}\right)\right)\right|=K,
$$

for some $K>0$ that is independent of $k$ and for which $\delta_{k} \rightarrow 0$ as $k \rightarrow \infty$. (Otherwise we could obtain a contradiction to Lemma 9 by integrating over $\left[z_{-1}^{k}, z_{1}^{k}\right]$, given that without loss of generality we can assume $g$ is of one sign over this interval.)

Now, by the mean value theorem, the bound (2.3) and (2.10), it follows that

$$
\left|u_{k}\left(z_{-1}^{k}\right)-u_{k}\left(z_{1}^{k}\right)\right| \leq \Theta \delta_{k}
$$

where here and below $\Theta$ is some, possibly different, constant independent of $k$. Moreover, by the monotonicity of $u_{k}$, it follows that

$$
u_{k}\left(z_{-1}^{k}\right)<u_{k}\left(z_{0}^{k}\right)<u_{k}\left(z_{1}^{k}\right) .
$$


The only way that (2.10)-(2.12) does not provide a contradiction is if

$$
\left|v_{k}\left(z_{-1}^{k}\right)-\hat{\gamma}\left(u_{k}\left(z_{-1}^{k}\right)\right)\right|<\Theta \delta_{k} \quad \text { and } \quad\left|v_{k}\left(z_{1}^{k}\right)\right|<\Theta \delta_{k} .
$$

As for each $k,\left(u_{k}, v_{k}\right)$ is a component-wise monotone function on $\mathcal{D}$, there can be at most one such "jump" in the solution i.e. for each fixed $k$ sufficiently large,

$$
\sup _{z \in \mathcal{D} \mid z<z_{0}^{k}-\frac{1}{k}}\left|v_{k}(z)-\hat{\gamma}\left(u_{k}(z)\right)\right|<\Theta \delta_{k} \quad \text { and } \sup _{z \in \mathcal{D} \mid z>z_{0}^{k}+\frac{1}{k}}\left|v_{k}(z)\right|<\Theta \delta_{k} .
$$

Also, by construction, $z_{0}^{k}$ tends to some $z^{*} \in \mathcal{D}$ as $k \rightarrow \infty$ and so for all $k$ sufficiently large, we have

$$
\sup _{z \in \mathcal{D} \backslash \Xi_{k}}\left|g\left(u_{k}(z), v_{k}(z)\right)\right|<\Theta \delta_{k},
$$

where $\Xi_{k}=\mathcal{B}_{\frac{2}{k}}\left(z^{*}\right) \bigcap \mathcal{D}$. Finally, the monotonicity of $\left(u_{k}, v_{k}\right)$ on $\mathbb{R}$ ensures that if such a jump occurs in a closed interval $\mathcal{D}$, then this jump is unique in any closed interval of $\mathbb{R}$ containing $\mathcal{D}$ and the result is proved.

Lemma 11. Let $\mathcal{D}$ be any closed interval in $\mathbb{R} \backslash z^{*}$ with $z^{*}$ as defined in Lemma 10. Then $\exists v^{*} \in C^{0}(\mathcal{D})$ and a subsequence $\left\{v_{k_{l}}\right\}_{l=1}^{\infty}$ of $\left\{v_{k}\right\}_{k=1}^{\infty}$ such that

$$
v_{k_{l}} \rightarrow v^{*} \text { in } C^{0}(\mathcal{D}) \text {. }
$$

Proof. By the arguments given in the proof of Lemma 10, on any closed interval $\mathcal{D}$ of $\mathbb{R} \backslash z^{*}$ either $v_{k} \rightarrow \hat{\gamma}\left(u^{*}\right)$ or $v_{k} \rightarrow 0$ uniformly as $k \rightarrow \infty$ and the result follows.

Finally we can complete the proof of Theorem 4.

Proof of Theorem 4 (ii). For any closed interval $\mathcal{D} \subset \mathbb{R} \backslash z^{*}$, it follows by Lemmas 8, 10 and 11 , that $\left(u_{k}, v_{k}\right) \rightarrow\left(u^{*}, v^{*}\right)$ uniformly on $\mathcal{D}$ and that $g\left(u^{*}(z), v^{*}(z)\right)=0 \forall z \in \mathcal{D}$. From the $u$-equation in (1.3) and using similar arguments to those above, it follows that $u_{k} \rightarrow u^{*}$ in $C^{2}(\mathcal{D})$. Consequently, the limit $\left(u^{*}, v^{*}\right)$ satisfies

$$
0=u^{*^{\prime \prime}}+f\left(u^{*}, v^{*}\right), \quad 0=g\left(u^{*}, v^{*}\right),
$$

on $\mathcal{D}$. Finally and as above, we may apply similar arguments to those in the proof of Theorem 2.1 in [6] to ensure that $\left(u^{*}, v^{*}\right)=\left(u_{0}, v_{0}\right)$.

We end this section by revisiting the method of obtaining bounds on $\left|v_{k}\right|_{1}$ used in [10]. Essentially, for a sub-class of problems, bounds on $\left|v^{\prime}(z)\right|$ can be obtained independent of the wave speed $c$, irrespective of its limiting value.

Lemma 12. Let $(u, v)$ be a solution of (1.3) and suppose

$$
g_{v}(u(z), v(z))<-C, \quad z \in \mathbb{R},
$$

for some constant $C>0$. Then there exists a constant $K>0$ such that

$$
\left|v^{\prime}(z)\right| \leq K, \quad \forall z \in \mathbb{R} .
$$


Proof. Suppose that the maximum value of $\left|v^{\prime}(z)\right|$ in $\mathbb{R}$ occurs at $z=z_{0}$. Then

$$
v^{\prime \prime}\left(z_{0}\right)=0 \quad \text { and } \quad v^{\prime \prime \prime}\left(z_{0}\right) \geq 0 .
$$

Now differentiating the second equation of (1.3) gives

$$
\epsilon^{2} v^{\prime \prime \prime}+c v^{\prime \prime}+g_{u}(u, v) u^{\prime}+g_{v}(u, v) v^{\prime}=0 .
$$

By evaluating this equation at $z_{0}$ and by noting the signs of each of the terms in this equation, it follows that

$$
\left|g_{v}\left(u\left(z_{0}\right), v\left(z_{0}\right)\right) v^{\prime}\left(z_{0}\right)\right| \leq\left|g_{u}\left(u\left(z_{0}\right), v\left(z_{0}\right)\right) u^{\prime}\left(z_{0}\right)\right| \text {. }
$$

That is

$$
\left|v^{\prime}\left(z_{0}\right)\right| \leq \frac{1}{C}\left|g_{u}\left(u\left(z_{0}\right), v\left(z_{0}\right)\right) u^{\prime}\left(z_{0}\right)\right|<K
$$

for some constant $K$, by (2.13), the smoothness of $g_{u}$ and the bounds on $|u|_{1}$ given in Lemmas 6 and 8 .

Remark 13. For the CLV model, it follows from a standard phase-plane analysis, that in the case where the wave speed $c<0$, solution trajectories lie in the region of $(u, v)$-space where $g(u, v)<0$. For this model,

$$
g(u, v)<0 \quad \Rightarrow \quad g_{v}(u, v)<-C,
$$

and so Lemma 12 can be applied directly in this case. However, the calculations in the next section reveal that if $c_{k}<0$, then $\lim _{k \rightarrow \infty} c_{k}=c_{0}<0$. Therefore, this bound provides no further information to that given in Lemma 6. Whether Lemma 12 offers an advantage over Lemma 6 for any realistic competition model of the kind discussed here remains an open question.

\section{Computing the sign of the wave speed}

As detailed in the Introduction, when $\epsilon>0$, (1.7) does not form a free energy function for (1.3). We now construct a suitable alternative and use it to determine the direction of the travelling wave solutions in the general case.

We begin by assuming the existence of a suitable energy function for (1.3) in order to demonstrate how it relates to the wave direction.

Lemma 14. Fix $\epsilon>0$. Let $(u, v, c)$ be the corresponding monotone solution of (1.3). Let

$$
F_{\epsilon}(u, v):=-\frac{1}{2}\left(u^{\prime}\right)^{2}-\frac{1}{2}\left(\epsilon v^{\prime}\right)^{2}+H_{\epsilon}(u, v)
$$

where

$$
\nabla H_{\epsilon}(u, v)=(-f(u, v),-g(u, v))^{T} .
$$

Then

$$
\operatorname{sign}\left(\frac{d}{d z} F_{\epsilon}\right)=\operatorname{sign}\left(H_{\epsilon}\left(S_{+}\right)-H_{\epsilon}\left(S_{-}\right)\right)=\operatorname{sign}(c) .
$$


Proof. The proof is straightforward. By differentiating $F_{\epsilon}$ wrt $z$ and using (1.3), it follows that

$$
\begin{aligned}
F_{\epsilon}^{\prime}(u, v) & =-u^{\prime} u^{\prime \prime}-\epsilon^{2} v^{\prime} v^{\prime \prime}+\left(H_{\epsilon}\right)_{u} u^{\prime}+\left(H_{\epsilon}\right)_{v} v^{\prime} \\
& =-u^{\prime}\left(u^{\prime \prime}+f(u, v)\right)-v^{\prime}\left(\epsilon^{2} v^{\prime \prime}+g(u, v)\right) \\
& =c\left(\left(u^{\prime}\right)^{2}+\left(v^{\prime}\right)^{2}\right)
\end{aligned}
$$

Therefore,

$$
\begin{aligned}
c \int_{\mathbb{R}}\left(\left(u^{\prime}\right)^{2}+\left(v^{\prime}\right)^{2}\right) d z & =\int_{\mathbb{R}} F_{\epsilon}^{\prime}(u, v) d z \\
& =\left.F_{\epsilon}(u(z), v(z))\right|_{-\infty} ^{\infty}=H_{\epsilon}\left(S_{+}\right)-H_{\epsilon}\left(S_{-}\right)
\end{aligned}
$$

and the results follow directly.

The next result determines a class of functions that satisfy the required conditions on $H_{\epsilon}$. For each fixed $\epsilon$, define $P_{\epsilon}:[0,1] \rightarrow[0,1]$ be the one-to-one function defined by $P_{\epsilon}(1)=0, P_{\epsilon}(0)=1$, $P_{\epsilon}(u(z))=v(z), z \in \mathbb{R}$, where $(u(z), v(z), c)$ is the monotone solution of (1.3). (That is $v=P_{\epsilon}(u)$ is the unique monotone solution trajectory in the $(u, v)$-plane, parameterized by $z$ and joining $(0,1)$ with $(1,0)$, which represents the travelling wave solution with speed $c$.) For ease of exposition, let $\left(P_{\epsilon}\right)^{-1} \equiv Q_{\epsilon}$. Note that the convergence results given in Theorem 4 ensure that for $\epsilon$ sufficiently small, the solution path $v=P_{\epsilon}(u)$ is close to the solution path $v=P_{0}(u)$ for the degenerate problem (1.6). To assist the subsequent discussions, a schematic representation of this relationship and to the path $v=\gamma(u)$ is given in Figure 3. In the case $h\left(S_{+}\right)=0$, all three paths coincide.

(a)

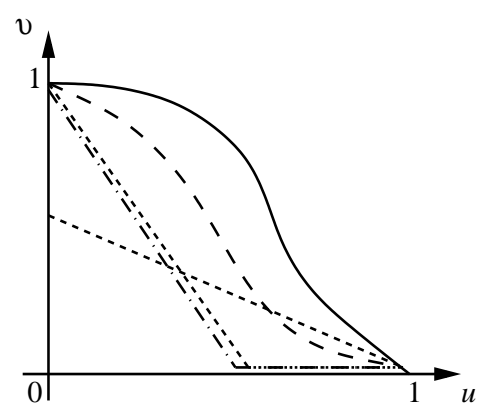

(b)

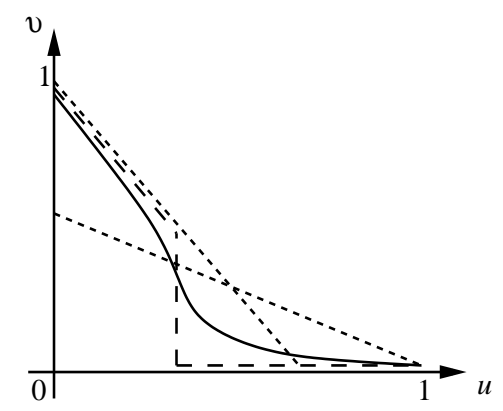

Figure 3: Schematic representation of the solutions paths $v=P_{\epsilon}(u)$ (solid line) and $v=P_{0}(u)$ (dashed line) in the cases (a) $h\left(S_{+}\right)<0$; (b) $h\left(S_{+}\right)>0$. The nullclines (dotted lines) are shown as straight lines for ease of visualisation and the path $v=\gamma(u)$ is shown in (a) (dot-dashed line). 
Let $(\phi, \psi) \in\left[C^{2}(\mathbb{R})\right]^{2},(\phi, \psi): \mathbb{R} \rightarrow[0,1]^{2}$ and for each fixed $\epsilon$, define $H_{\epsilon}(\phi, \psi)$ by

$$
\begin{aligned}
{\left[H_{\epsilon}(\phi, \psi)\right](z)=} & -\int_{0}^{\phi(z)} f(\sigma, \psi(z)) d \sigma-\int_{1}^{\psi(z)} g(\phi(z), \tau) d \tau \\
& -\int_{\psi(z)}^{1} \int_{0}^{Q_{\epsilon}(\tau)} f_{v}(\sigma, \tau) d \sigma d \tau+\int_{0}^{\phi(z)} \int_{1}^{P_{\epsilon}(\sigma)} g_{u}(\sigma, \tau) d \tau d \sigma .
\end{aligned}
$$

Lemma 15. The function $H_{\epsilon}$ defined in (3.3) satisfies the condition (3.2) required in Lemma 14. Moreover,

$$
\left[H_{\epsilon}\left(S_{+}\right)\right](z) \equiv H_{\epsilon}\left(S_{+}\right)=-\int_{0}^{1} f\left(\sigma, P_{\epsilon}(\sigma)\right) d \sigma+\int_{0}^{1} g\left(Q_{\epsilon}(\tau), \tau\right) d \tau
$$

and $H_{\epsilon}\left(S_{-}\right)=0$ for all $\epsilon>0$.

Proof. It follows directly from (3.3), that

$$
\frac{\partial}{\partial \phi}\left(H_{\epsilon}(\phi, \psi)\right)=-f(\phi, \psi)-\int_{1}^{\psi} g_{u}(\phi, \tau) d \tau+\int_{1}^{P_{\epsilon}(\phi)} g_{u}(\phi, \tau) d \tau .
$$

If $(\phi, \psi)=(u, v)$, the monotone solution of (1.3), then the first component of (3.2) follows from the identity $v=P_{\epsilon}(u)$. Also,

$$
\frac{\partial}{\partial \psi}\left(H_{\epsilon}(\phi, \psi)\right)=-\int_{0}^{\phi} f_{v}(\sigma, \psi) d \sigma-g(\phi, \psi)+\int_{0}^{Q_{\epsilon}(\psi)} f_{v}(\sigma, \psi) d \sigma
$$

Again, if $(\phi, \psi)=(u, v)$, the monotone solution of (1.3), then the second component of (3.2) follows from the identity $u=Q_{\epsilon}(v)$. Next,

$$
\begin{aligned}
{\left[H_{\epsilon}\left(S_{+}\right)\right](z) \equiv H_{\epsilon}(1,0)=} & -\int_{0}^{1} f(\sigma, 0) d \sigma-\int_{1}^{0} g(1, \tau) d \tau \\
& -\int_{0}^{1} \int_{0}^{Q_{\epsilon}(\tau)} f_{v}(\sigma, \tau) d \sigma d \tau+\int_{0}^{1} \int_{1}^{P_{\epsilon}(\sigma)} g_{u}(\sigma, \tau) d \tau d \sigma
\end{aligned}
$$

By changing the order of integration and recalling that $\left(P_{\epsilon}\right)^{-1}=Q_{\epsilon}$, it follows that

$$
\begin{aligned}
H_{\epsilon}(1,0)= & -\int_{0}^{1} f(\sigma, 0) d \sigma-\int_{1}^{0} g(1, \tau) d \tau \\
& -\int_{0}^{1} f\left(\sigma, P_{\epsilon}(\sigma)\right)-f(\sigma, 0) d \sigma+\int_{1}^{0} g(1, \tau)-g\left(Q_{\epsilon}(\tau), \tau\right) d \tau \\
= & -\int_{0}^{1} f\left(\sigma, P_{\epsilon}(\sigma)\right) d \sigma+\int_{0}^{1} g\left(Q_{\epsilon}(\tau), \tau\right) d \tau
\end{aligned}
$$

as required.

Finally, $H_{\epsilon}\left(S_{-}\right)=0$ follows directly from the definition of $H_{\epsilon}$. This scaling is obtained without loss of generality. 


\section{Lemma 16.}

$$
\operatorname{sign}\left(-\int_{0}^{1} f\left(\sigma, P_{\epsilon}(\sigma)\right) d \sigma\right)=\operatorname{sign}\left(\int_{0}^{1} g\left(Q_{\epsilon}(\tau), \tau\right) d \tau\right)=\operatorname{sign}(c) .
$$

Proof. Multiplying the first and second equations in (1.3) by $u^{\prime}$ and $v^{\prime}$, respectively and integrating over $\mathbb{R}$ yields

$$
-c \int_{\mathbb{R}}\left(u^{\prime}\right)^{2} d z=\int_{\mathbb{R}} u^{\prime \prime} u^{\prime} d z+\int_{\mathbb{R}} f(u, v) u^{\prime} d z=\int_{0}^{1} f\left(\sigma, P_{\epsilon}(\sigma)\right) d \sigma
$$

and

$$
-c \int_{\mathbb{R}}\left(v^{\prime}\right)^{2} d z=\int_{\mathbb{R}} \epsilon^{2} v^{\prime \prime} v^{\prime} d z+\int_{\mathbb{R}} g(u, v) v^{\prime} d z=\int_{1}^{0} g\left(Q_{\epsilon}(\tau), \tau\right) d \tau .
$$

The result follows immediately.

These results can be summarised as follows.

Theorem 17. For each fixed $\epsilon>0$, let $\left(u_{\epsilon}, v_{\epsilon}, c_{\epsilon}\right)$ be the corresponding monotone solution of (1.3). Let $H_{\epsilon}(\phi, \psi)$ be defined as in (3.3). Then

$$
H_{\epsilon}\left(S_{+}\right)>0 \Longleftrightarrow c_{\epsilon}>0, \quad H_{\epsilon}\left(S_{+}\right)<0 \Longleftrightarrow c_{\epsilon}<0, \quad H_{\epsilon}\left(S_{+}\right)=0 \Longleftrightarrow c_{\epsilon}=0 .
$$

Remark 18. The Maxwell Curve. It is clear that $H_{\epsilon}\left(S_{+}\right)=H_{\epsilon}\left(S_{+}, \boldsymbol{\Lambda}\right)$ where $\boldsymbol{\Lambda}$ is the vector of the system parameters. With the functional forms fixed, $H_{\epsilon}\left(S_{+}, \boldsymbol{\Lambda}\right)=0$ defines a relationship between $\epsilon$ and $\Lambda$. Under certain genericity conditions, this defines a curve in the $\epsilon-\Lambda$ plane. We call this the Maxwell Curve for (1.1), following the definition of the Maxwell Curve for 4th order problems see e.g. [12] and the references therein.

Remark 19. The definition of the potential $H_{\epsilon}$ gives a formal way of determining the wave speed, although it is an implicit function of the solution itself. It would seem then that to compute the wave speed for any given problem, would therefore require (at least an approximate) evaluation of the solution. However, using the definition of the potential given in [6], the definition introduced here in (3.3) and the limiting arguments given above, we now show that the sign of the wave speed can be computed directly, at least for the case $\epsilon$ sufficiently small but non-zero, using only information related to the functions $f$ and $g$. This result is particularly powerful in the case where $c_{0}=0$, i.e. the wave speed of the degenerate problem is zero. In this case, the sign of the wave speed for the $\epsilon$-small case is entirely unclear. Indeed, in the final section, where non-monotone functions $g$ are considered, we show that in this case, the $\epsilon$-small wave speed can be either positive or negative.

Theorem 20. Let $H_{\epsilon}\left(S_{+}\right)$be given as in (3.4). Then there exists a number $H_{0}\left(S_{+}\right)$such that $\lim _{\epsilon \rightarrow 0} H_{\epsilon}\left(S_{+}\right)=H_{0}\left(S_{+}\right)$but $H_{0}\left(S_{+}\right) \neq h\left(S_{+}\right)$in general. However,

(i) $h\left(S_{+}\right)<0 \Longrightarrow H_{0}\left(S_{+}\right)<0$,

(ii) $h\left(S_{+}\right)=0 \Longrightarrow H_{0}\left(S_{+}\right)=0$,

(iii) $h\left(S_{+}\right)>0 \Longrightarrow H_{0}\left(S_{+}\right)>0$. 
Proof. (i) If $h\left(S_{+}\right)<0$, then Theorem 3 (i) ensures $c_{0}<0$ and thus that $g\left(u_{0}, v_{0}\right)<0$ (apart from the end points where $\left.g\left(S_{ \pm}\right)=0\right)$. By Theorem 4 it follows that $f\left(\cdot, P_{\epsilon}(\cdot)\right) \rightarrow f\left(\cdot, P_{0}(\cdot)\right)$ and $g\left(Q_{\epsilon}(\cdot), \cdot\right) \rightarrow g\left(Q_{0}(\cdot), \cdot\right)$ uniformly on $[0,1] \times[0,1]$ as $\epsilon \rightarrow 0$, where $v=P_{0}(u)$ (alt. $u=Q_{0}(v)$ ) is the trajectory in the $(u, v)$-plane representing the solution $\left(u_{0}, v_{0}\right)$ to (1.6). Hence, we may pass directly to the limit in (3.4) to yield

$$
\begin{aligned}
\lim _{\epsilon \rightarrow 0} H_{\epsilon}\left(S_{+}\right) & =\lim _{\epsilon \rightarrow 0}\left(-\int_{0}^{1} f\left(\sigma, P_{\epsilon}(\sigma)\right) d \sigma+\int_{0}^{1} g\left(Q_{\epsilon}(\tau), \tau\right) d \tau\right) \\
& =-\int_{0}^{1} f\left(\sigma, P_{0}(\sigma)\right) d \sigma+\int_{0}^{1} g\left(Q_{0}(\tau), \tau\right) d \tau=: H_{0}\left(S_{+}\right) .
\end{aligned}
$$

From Lemma 16 and as $g\left(Q_{0}(\tau), \tau\right)<0, \tau \in(0,1)$, it follows that $H_{0}\left(S_{+}\right)<0$.

(ii) If $h\left(S_{+}\right)=0$, then by Theorem 3 (ii) the solution $v_{0}$ is continuous at $z^{*}$ as defined above but not differentiable. Moreover, in this case $c_{0}=0$ and the solution path is defined by $g\left(Q_{0}(\tau), \tau\right)=0$ for $\tau \in[0,1]$. Hence, arguing as above, we may pass directly to the limit in (3.4) and $H_{0}\left(S_{+}\right)=0$.

(iii) Finally, if $h\left(S_{+}\right)>0$, then from Theorem 3 (ii), $v_{0}$ has a unique discontinuity at $z=z^{*}$. Hence, from (3.4), and for any $\delta>0$,

$$
\begin{aligned}
\lim _{\epsilon \rightarrow 0} H_{\epsilon}\left(S_{+}\right)= & \lim _{\epsilon \rightarrow 0}\left(-\int_{0}^{1} f\left(\sigma, P_{\epsilon}(\sigma)\right) d \sigma+\int_{0}^{1} g\left(Q_{\epsilon}(\tau), \tau\right) d \tau\right) \\
= & -\lim _{\epsilon \rightarrow 0}\left(\int_{0}^{u^{*}-\delta}+\int_{u^{*}-\delta}^{u^{*}+\delta}+\int_{u^{*}+\delta}^{1} f\left(\sigma, P_{\epsilon}(\sigma)\right) d \sigma\right) \\
& +\lim _{\epsilon \rightarrow 0}\left(\int_{0}^{\delta}+\int_{\delta}^{v^{*}-\delta}+\int_{v^{*}-\delta}^{v^{*}+\delta}+\int_{v^{*}+\delta}^{1} g\left(Q_{\epsilon}(\tau), \tau\right) d \tau\right),
\end{aligned}
$$

where $\left(u^{*}, v^{*}\right)=\left(u_{0}\left(z^{*}\right), v_{0}\left(z^{*}\right)\right)$. The uniformity of the limit away from $z=z^{*}$ ensures that

$$
\begin{aligned}
& -\lim _{\epsilon \rightarrow 0}\left(\int_{0}^{u^{*}-\delta}+\int_{u^{*}+\delta}^{1} f\left(\sigma, P_{\epsilon}(\sigma)\right) d \sigma\right)+\lim _{\epsilon \rightarrow 0}\left(\int_{v^{*}+\delta}^{1} g\left(Q_{\epsilon}(\tau), \tau\right) d \tau\right) \\
& =-\left(\int_{0}^{u^{*}-\delta}+\int_{u^{*}+\delta}^{1} \lim _{\epsilon \rightarrow 0} f\left(\sigma, P_{\epsilon}(\sigma)\right) d \sigma\right)+\left(\int_{v^{*}+\delta}^{1} \lim _{\epsilon \rightarrow 0} g\left(Q_{\epsilon}(\tau), \tau\right) d \tau\right) \\
& =-\left(\int_{0}^{u^{*}-\delta}+\int_{u^{*}+\delta}^{1} f\left(\sigma, P_{0}(\sigma)\right) d \sigma\right)+\left(\int_{v^{*}+\delta}^{1} g\left(Q_{0}(\tau), \tau\right) d \tau\right) .
\end{aligned}
$$

This holds for any $\delta>0$ and all integrals are well-defined. Hence, on taking the limit as $\delta \rightarrow 0$, (3.6) equates to

$$
-\int_{0}^{1} f\left(\sigma, P_{0}(\sigma)\right) d \sigma+\int_{v^{*}}^{1} g\left(Q_{0}(\tau), \tau\right) d \tau
$$

However, from the proof of Theorem 2.1 in [5], it follows directly that,

$$
-\int_{0}^{1} f\left(\sigma, P_{0}(\sigma)\right) d \sigma=0 .
$$


Moreover, on $\left[v^{*}, 1\right], Q_{0}(v)=\hat{\gamma}(v)$ and hence $g\left(Q_{0}(\tau), \tau\right)=0$. Therefore, (3.7) equates to zero.

Also,

$$
\left|\left(\int_{u^{*}-\delta}^{u^{*}+\delta} f\left(\sigma, P_{\epsilon}(\sigma)\right) d \sigma\right)\right| \leq 2 \delta \max _{(\sigma, \tau) \in[0,1]^{2}}|f(\sigma, \tau)|,
$$

and

$$
\left|\left(\int_{0}^{\delta}+\int_{v^{*}-\delta}^{v^{*}+\delta} g\left(Q_{\epsilon}(\tau), \tau\right) d \tau\right)\right| \leq 3 \delta \max _{(\sigma, \tau) \in[0,1]^{2}}|g(\sigma, \tau)| .
$$

These bounds are independent of $\epsilon$ and consequently the limits (as $\epsilon \rightarrow 0$ ) of these integrals tend to zero on taking the limit as $\delta \rightarrow 0$. Hence, for any $\delta>0$, we have from (3.5) that

$$
\lim _{\epsilon \rightarrow 0} H_{\epsilon}\left(S_{+}\right)=\lim _{\epsilon \rightarrow 0} \int_{\delta}^{v^{*}-\delta} g\left(Q_{\epsilon}(\tau), \tau\right) d \tau
$$

But for any $\delta>0, Q_{\epsilon}(\cdot) \rightarrow u^{*}$ uniformly in $\left[\delta, v^{*}-\delta\right]$ and hence $g\left(Q_{\epsilon}(\cdot), \cdot\right)$ converges uniformly on $\left[\delta, v^{*}-\delta\right]$ to $g\left(u^{*}, \cdot\right)$. Hence, on taking the limit as $\delta \rightarrow 0$, yields

$$
\lim _{\epsilon \rightarrow 0} H_{\epsilon}\left(S_{+}\right)=\int_{0}^{v^{*}} g\left(u^{*}, \tau\right) d \tau=: H_{0}\left(S_{+}\right) .
$$

However,

$$
\int_{0}^{v^{*}} g\left(u^{*}, \tau\right) d \tau>0
$$

because the path $u=u^{*}, v \in\left[0, v^{*}\right]$ lies below the $g$ nullcline in the $(u, v)$-plane and hence $g>0$ by Assumption 1. Thus $H_{0}\left(S_{+}\right)>0$ in this case.

Finally, recall that the curves in the $(u, v)$-plane described by $v=P_{0}(u)$ and $v=\gamma(u)$ are not the same in general (see Figure $3(\mathrm{~b})$ ). Hence, $H_{0}\left(S_{+}\right) \neq h\left(S_{+}\right)$apart from when they are both zero. This completes the proof.

Remark 21. From Theorem 3 it follows that $h\left(S_{+}\right)>0$ implies that $c_{0}=0$. Also, Theorem 20 states that $h\left(S_{+}\right)>0 \Longrightarrow H_{0}\left(S_{+}\right)>0$ and from Theorem 17 (and Lemma 16) we would therefore expect $c_{0}>0$. This apparent contradiction can be resolved by reconsidering the proof of Lemma 16, which is only true for each fixed $\epsilon>0$. On taking the limit as $\epsilon \rightarrow 0$, we need to reconsider the boundedness of all terms. The following result clarifies the situation and leads to a better understanding of the formation of the singularity in the v-component of the solution.

Lemma 22. If $h\left(S_{+}\right)>0$, then $c_{\epsilon} \rightarrow 0$ and $\int_{\mathbb{R}}\left(v_{\epsilon}^{\prime}\right)^{2} d z \rightarrow \infty$ as $\epsilon \rightarrow 0$.

Proof. Similar to the proof of Lemma 16, from the $u$-equation in (1.3), it follows that

$$
c_{\epsilon} \int_{\mathbb{R}}\left(u_{\epsilon}^{\prime}\right)^{2} d z=-\int_{0}^{1} f\left(\sigma, P_{\epsilon}(\sigma)\right) d \sigma .
$$


As detailed in the proof of Theorem 20, it follows that if $h\left(S_{+}\right)>0$ then the right hand side tends to zero as $\epsilon \rightarrow 0$. Also, by (2.3), and following arguments similar to those in the proof of Lemma 7, for any closed interval $D \subset \mathbb{R} \backslash z^{*}$,

$$
\int_{\mathbb{R}}\left(u_{\epsilon}^{\prime}\right)^{2} d z \geq \int_{D}\left(u_{\epsilon}^{\prime}\right)^{2} d z \rightarrow \int_{D}\left(u_{0}^{\prime}\right)^{2} d z>0
$$

with positivity following directly from the construction of $u_{0}$. This implies $c_{\epsilon} \rightarrow 0$. Moreover, from the $v$-equation in (1.3), it follows that

$$
c_{\epsilon} \int_{\mathbb{R}}\left(v_{\epsilon}^{\prime}\right)^{2} d z=\int_{0}^{1} g\left(Q_{\epsilon}(\tau), \tau\right) d \tau .
$$

Again, the proof of Theorem 20 reveals that the limit of the right hand side is strictly positive. Thus

$$
\int_{\mathbb{R}}\left(v_{\epsilon}^{\prime}\right)^{2} d z>\frac{K}{c_{\epsilon}}
$$

for some $K>0$, independent of $\epsilon$. The result follows on taking the limit as $\epsilon \rightarrow 0$.

Finally, the above results can be summarised as follows. This theorem links the energy function derived in [6] for the reduced problem (1.6) to the direction of travelling waves in the general problem (1.3), at least for $\epsilon$ sufficiently small. Recall that given any problem of the form (1.3), the energy function $h\left(S_{+}\right)$can be computed directly from the kinetics and does not require a priori knowledge of the solutions.

Theorem 23. Let $\left(u_{\epsilon}, v_{\epsilon}, c_{\epsilon}\right)$ be a component-wise monotone solution of (1.3).

(i) If $h\left(S_{+}\right)<0$ then for $\epsilon$ sufficiently small, $c_{\epsilon}<0$ and $c_{\epsilon} \rightarrow c_{0}<0$ as $\epsilon \rightarrow 0$.

(ii) If $h\left(S_{+}\right)>0$ then for $\epsilon$ sufficiently small $c_{\epsilon}>0$ and $c_{\epsilon} \rightarrow 0$ as $\epsilon \rightarrow 0$, and $\lim _{\epsilon \rightarrow 0}\left\|v_{\epsilon}^{\prime}\right\|_{L^{2}(\mathbb{R})}=\infty$.

Remark 24. For the CLV problem it is straightforward to show that $h\left(S_{+}\right)=\left(\alpha-\beta^{2}\right) / 6 \beta^{2}$. Hence, for $\epsilon$ sufficiently small, the wave speed is determined by a simple combination of the competition rate parameters. Where $h\left(S_{+}\right)=0$, numerical integration suggest that $H_{\epsilon}\left(S_{+}\right)>0$ and hence $c_{\epsilon}>0$ for $0<\epsilon<<1$. However, a proof of this result has yet to be obtained and remains an open problem.

\section{Non-monotone nullclines}

In this final section we relax condition (4) of Assumption 1 to include a class of non-monotone functions $g$. In particular, we replace (4) by

$\left(4^{*}\right)$ The non-trivial solutions $(u, v)$ of $g(u, v)=0$ are given by $u=\Gamma(v)$, for a continuous 
function $\Gamma:[0,1] \rightarrow[0, \infty)$ with $\Gamma(1)=0$ and $\Gamma(0)=\hat{u}$, where $0<\hat{u}<1$. Moreover, $\Gamma^{\prime}(v)=0$ implies $\Gamma$ has a local maximum at $v$.

This is the case considered in [6] and we follow the constructions given there. The assumptions on $f$ are unchanged (note that $f$ could also be similarly non-monotone). A sketch of a typical $g$ nullcline satisfying $\left(4^{*}\right)$ is given in Figure 4 , where the points $u_{1}$ and $v_{1}$ are identified by

$$
u_{1}=\min \left\{1, \sup _{\tau \in(0,1)} \Gamma(\tau)\right\}, \quad v_{1}:=\gamma_{m}\left(u_{1}\right),
$$

where $\gamma_{m}$ is the unique maximal inverse of $\Gamma$, i.e. $\gamma_{m}(0)=1$ and

$$
\gamma_{m}(\sigma):=\max _{\tau \in(0,1)}\{\Gamma(\tau)=\sigma\}
$$

Note that $\gamma_{m}$ is monotone decreasing on $\left(0, u_{1}\right)$.

Clearly, functions that satisfy $\left(4^{*}\right)$ also satisfy $(4)$. As the latter case has already been covered, in the following we focus on kinetics for which $\Gamma$ is non-monotone, i.e. $u_{1}>\hat{u}$.

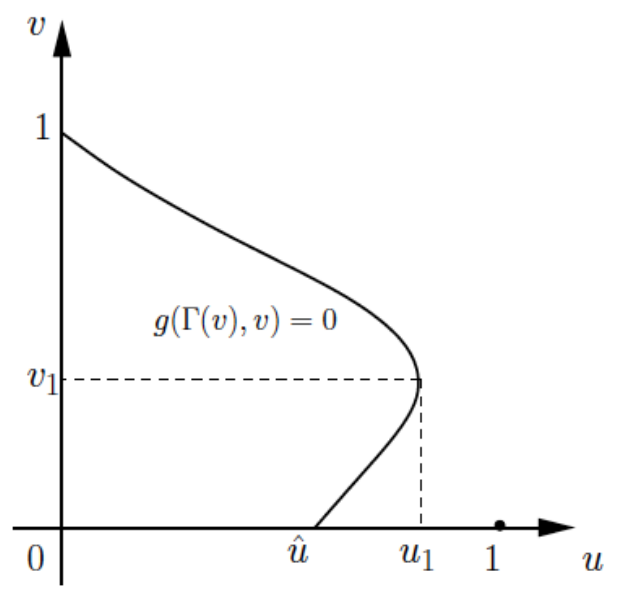

Figure 4: Schematic representation of the nullcline $g$ satisfying Assumption 1 with (4) replaced by $\left(4^{*}\right)$. The maximum of $\Gamma$ occurs at $v_{1}$ with $u_{1}=\Gamma\left(v_{1}\right)>\hat{u}$.

In [6] a functional $A(u)$ is defined by

$$
\begin{aligned}
A(u) & :=h(1,0)-h(u, 0)+h\left(u, \gamma_{m}(u)\right) \\
& =-\int_{0}^{u} f\left(\sigma, \gamma_{m}(\sigma)\right) d \sigma-\int_{u}^{1} f(\sigma, 0) d \sigma .
\end{aligned}
$$

If $u_{1}=1$, then

$$
A(1)=A\left(u_{1}\right)=-\int_{0}^{1} f\left(\sigma, \gamma_{m}(\sigma)\right) d \sigma>0
$$


With $\left(4^{*}\right)$ replacing (4), all the results in Theorem 3 hold with $A\left(u_{1}\right)$ replacing $h\left(S_{+}\right)$. (Note that under condition (4), $u_{1}=\hat{u}, v_{1}=0$ and $A\left(u_{1}\right)=h(1,0)=h\left(S_{+}\right)$.) Thus in the case $A\left(u_{1}\right)<0$, the solution $\left(u_{0}, v_{0}, c_{0}\right)$ is classical and $c_{0}<0$. In the case $A\left(u_{1}\right) \geq 0$, the solution $\left(u_{0}, v_{0}, c_{0}\right)$ has $c_{0}=0$ and a unique discontinuity at $\left(u_{0}, v_{0}\right)\left(z^{*}\right)=\left(u^{*}, v^{*}\right)$, with $u^{*} \leq u_{1}$ for $u_{1}<1$. The value $u^{*}$ is the unique positive solution to $A\left(u^{*}\right)=0$. Finally, as shown in [6], $A^{\prime}(u)>0$.

It is straightforward to show that the convergence results proved in Theorem 4 here also hold with $\left(4^{*}\right)$ replacing $(4)$. (Lemmas 6-9 follow without alteration. Lemma 10 requires extra cases to be considered but the method of proof and results are unaltered.) Similarly, the definition of the energy function $H_{\epsilon}$ given in (3.4) and the relationship between $H_{\epsilon}\left(S_{+}\right)$and the wave speed $c_{\epsilon}$ as detailed in Theorem 17 are unaltered.

It remains to discuss the relationship between the energy function $H_{\epsilon}$ defined here and that defined in [6]. This result requires one further definition.

Definition 25. Define $\underline{u} \in(\hat{u}, 1]$ by $\underline{u}:=\min \{s, 1\}$ where $s$ is the unique solution of $G(s)=0$, where

$$
G(u):=\int_{0}^{\gamma_{m}(u)} g(u, \tau) d \tau
$$

Remark 26. It is clear by the definition of $\hat{u}$ and conditions (3) and $\left(4^{*}\right)$ in Assumption 1, that $G(\hat{u})>0$, and the existence of the unique value $\underline{u}$ follows by the continuity of $g$ and for $u \in\left(\hat{u}, u_{1}\right)$,

$$
\begin{aligned}
G^{\prime}(u) & =\gamma_{m}^{\prime}(u) g\left(u, \gamma_{m}(u)\right)+\int_{0}^{\gamma_{m}(u)} g_{u}(u, \tau) d \tau \\
& =\int_{0}^{\gamma_{m}(u)} g_{u}(u, \tau) d \tau<0 .
\end{aligned}
$$

Note also that the definition of $\underline{u}$ does not require a formal extension of the definition of $g$ for values of $s>1$. For if $G(1)>0$, then $\underline{u}=1$ and the value of $s$ is redundant.

We now have the following result that replaces Theorem 20 above.

Theorem 27. Suppose that Assumption 1 holds with (4) replaced by $\left(4^{*}\right)$. Let $H_{\epsilon}\left(S_{+}\right)$be given as in (3.4). Then there exists a number $H_{0}\left(S_{+}\right)$such that $\lim _{\epsilon \rightarrow 0} H_{\epsilon}\left(S_{+}\right)=H_{0}\left(S_{+}\right)$. Moreover,

(i) If $A(\underline{u})<0$ then $H_{0}\left(S_{+}\right)<0$,

(ii) If $A(\underline{u})=0$ then $H_{0}\left(S_{+}\right)=0$,

(iii) If $A(\underline{u})>0$ then $H_{0}\left(S_{+}\right)>0$.

Proof. Case $(i): A(\underline{u})<0$. We have two cases to consider. (a) Suppose $A\left(u_{1}\right) \geq 0$. Then as $A^{\prime}(u)>0$ and $A\left(u^{*}\right)=0$, by definition $\underline{u}<u^{*} \leq u_{1}$. Since $G(\underline{u})=0$ and $G^{\prime}(u)<0$, it follows that $G\left(u^{*}\right)<0$ and applying the arguments in the proof of Theorem 20 (ii) yields $H_{0}\left(S_{+}\right)<0$. (b) Suppose $A\left(u_{1}\right)<0$, then the arguments in the proof of Theorem 20 (i) can be applied unaltered and again $H_{0}\left(S_{+}\right)<0$

Case (ii): $A(\underline{u})=0$. Then $\underline{u}=u^{*}<u_{1}$ and $G\left(u^{*}\right)=0$. We can apply the arguments in the proof 
of Theorem 20 (ii) to yield $H_{0}\left(S_{+}\right)=0$.

Case (iii): $A(\underline{u})>0$. Then as $A^{\prime}(u)>0$, if follows that $\underline{\mathrm{u}}>u^{*}$ and thus $G\left(u^{*}\right)>0$, and again we can apply the arguments in the proof of Theorem 20 (ii) to yield $H_{0}\left(S_{+}\right)>0$.

In one easily-verified case, the sign of the limit is straight forward to compute. The proof follows from similar arguments to those used in Case (ii) above.

Corollary 28. If $G(1) \geq 0$ then $H_{0}\left(S_{+}\right)>0$.

Finally, we can relate the value of the energy function for the degenerate problem to the wave speed of the problem considered here. The proof follows directly from the arguments in the proof of Theorem 27.

Theorem 29. Let $\left(u_{\epsilon}, v_{\epsilon}, c_{\epsilon}\right)$ be a component-wise monotone solution of (1.3). If $\epsilon$ is sufficiently small and

(i) $A(\underline{u})<0 \leq A\left(u_{1}\right)$ then $c_{\epsilon}<0$ and $c_{\epsilon} \rightarrow 0$ as $\epsilon \rightarrow 0$.

(ii) $0<A(\underline{u}) \leq A\left(u_{1}\right)$ then $c_{\epsilon}>0$ and $c_{\epsilon} \rightarrow 0$ as $\epsilon \rightarrow 0$.

(iii) $A\left(u_{1}\right)<0$ then $c_{\epsilon}<0$ and $c_{\epsilon} \rightarrow c_{0}<0$ as $\epsilon \rightarrow 0$.

An alternative formulation to Theorem 29 that directly uses the definition of $G$ given above is as follows:

Theorem 30. Let $\left(u_{\epsilon}, v_{\epsilon}, c_{\epsilon}\right)$ be a component-wise monotone solution of (1.3). If $\epsilon$ is sufficiently small and

(i) $A\left(u_{1}\right) \geq 0$ and $G\left(u^{*}\right)>0$ then $c_{\epsilon}>0$ and $c_{\epsilon} \rightarrow 0$ as $\epsilon \rightarrow 0$.

(ii) $A\left(u_{1}\right) \geq 0$ and $G\left(u^{*}\right)<0$ then $c_{\epsilon}<0$ and $c_{\epsilon} \rightarrow 0$ as $\epsilon \rightarrow 0$.

(iii) $A\left(u_{1}\right)<0$ then $c_{\epsilon}<0 c_{\epsilon} \rightarrow c_{0}<0$ as $\epsilon \rightarrow 0$.

Remark 31. The values $\underline{u}, u_{1}$ and $u^{*}$ and thus the values $A(\underline{u}), A\left(u_{1}\right)$ and $G\left(u^{*}\right)$ can be directly computed using only the properties of the reaction terms $f$ and $g$. Thus, as with the monotone case, the sign of the wave speed of the full problem can be directly computed using only knowledge of these kinetics, at least for $\epsilon$ sufficiently small. However, as in the monotone case, the sign of the wave speed $c_{\epsilon}$ in the critical case $A(\underline{u})=0$ (alt. $\left.G\left(u^{*}\right)=0\right)$ remains an open question.

Remark 32. The function $F$ defined in (3.1) provides a measure of the energy associated with system (1.3). (A similar expression can be formulated for the system (1.1).) From the proof of Lemma 14, it follows that if $c_{\epsilon} \rightarrow 0$ as $\epsilon \rightarrow 0$ (i.e in the case where $v_{0}$ has a discontinuity at $\left.z=z^{*}\right)$, then $F_{\epsilon}^{\prime} \rightarrow 0$ pointwise on $\mathbb{R} \backslash z^{*}$. Therefore, as $\epsilon \rightarrow 0$, the total change in the energy $F$ (given by $H_{\epsilon}\left(S_{+}\right)$) over $\mathbb{R}$ is compressed to a discontinuous jump at the point $z^{*}$. The size of this discontinuity is equal to $H_{0}\left(S_{+}\right)=G\left(u^{*}\right)$.

\section{Acknowledgements}

EOA acknowledges the support of the Saudi Arabian Cultural Bureau. FAD acknowledges support from EPSRC grant EP/D043859. 


\section{References}

[1] R. S. Cantrell, C. Cosner. Spatial Ecology via Reaction-Diffusion Equations. John Wiley and Sons Ltd, New York, 2003.

[2] L.C. Evans. Partial Differential Equations, volume 19 of Graduate Studies in Mathematics. American Mathematical Society, Providence, Rhode Island, 2010.

[3] P. Grindrod. The Theory and Applications of Reaction-Diffusion Equations: Patterns and Waves. Clarendon Press, Oxford, 1996.

[4] J.S. Guo, J. Tsai. The asymptotic behavior of solutions of the buffered bistable system. J. Math. Biol., 53 (2006), No. 1, 179-213.

[5] S. Heinze, B. Schweizer. Creeping fronts in degenerate reaction-diffusion systems. Nonlinearity, 18 (2005), No. 6, 2455-2476.

[6] S. Heinze, B. Schweizer, H. Schwetlick. Existence of front solutions in degenerate reaction diffusion systems. Preprint 2004-03, SFB 359, University of Heidelberg, 2004.

[7] Y. Hosono. Singular perturbation analysis of travelling waves for diffusive Lotka-Volterra competition models. In Numerical and Applied Mathematics (Paris1989) IMACS Ann. Comput. Appl. Math., (1989), No 2., 687-692.

[8] Y. Hosono, M. Mimura. Singular perturbation approach to traveling waves in competing and diffusing species models. J. Math. Kyoto University, 22 (1982), No. 3, 435-461.

[9] B. Kazmierczak, V. Volpert. Travelling waves in partially degenerate reaction-diffusion systems. Mathematical Modelling of Natural Phenomena, 2 (2007), No. 2, 106-125.

[10] B. Kazmierczak, V. Volpert. Calcium waves in systems with immobile buffers as a limit of waves for systems with nonzero diffusion. Nonlinearity, 21 (2008), No. 1, 71-96.

[11] B. Kazmierczak, V. Volpert. Mechano-chemical calcium waves in systems with immobile buffers. Archives of Mechanics, 60 (2008), No. 1, 3-22.

[12] D.J. B. Lloyd, B. Sandstede, D. Avitabile, A.R. Champneys. Localized hexagon patterns of the planar Swift-Hohenberg equation. SIAM J. Appl. Dyn. Syst., 7 (2008), No. 3, 1049-1100.

[13] J.D. Murray. Mathematical Biology, II: Spatial Models and Biomedical Applications, volume 2. Springer-Verlag, Berlin, 2003.

[14] A. Okubo, S.A. Levin. Diffusion and Ecological Problems: Modern Perspectives. SpringerVerlag, New York, 2001.

[15] J.A. Smoller. Shock Waves and Reaction-Diffusion Equations. Springer-Verlag, Berlin, 1994. 
[16] J.C. Tsai, J. Sneyd. Existence and stability of traveling waves in buffered systems. SIAM J. Applied Math., 66 (2005), No. 1, 237-265.

[17] A.I. Volpert, V.A. Volpert, V.A. Volpert. Traveling Wave Solutions of Parabolic Systems: Translations of Mathematical Monographs, volume 140. American Mathematical Society, Providence, R.I., 1994. 